\title{
New Approach to Satellite Formation-Keeping: Exact Solution to the Full Nonlinear Problem
}

\author{
Hancheol $\mathrm{Cho}^{1}$ and $\mathrm{Adam} \mathrm{Yu}^{2}$
}

\begin{abstract}
This paper presents a new, simple, and exact solution to the formation keeping of satellites when the relative distance between the satellites is so large that the linearized relative equations of motion no longer hold. We employ a recently proposed approach, the Udwadia-Kalaba approach, which makes it possible to explicitly obtain the desired control function without making any approximations related to the nonlinearities in the underlying dynamics. We use an inertial frame of reference to describe the motion of a satellite and since no approximations are made, the results obtained apply to situations even when the distance between the satellites is arbitrarily large. The paper deals with a projected circular formation, but the methodology in this paper can be applied to any desired configuration or orbital requirements. Numerical simulations confirm the brevity and the accuracy of the analytical solution to the dynamical control problem developed herein.
\end{abstract}

DOI: 10.1061/(ASCE)AS.1943-5525.0000013

CE Database subject headings: Satellites; Orbits; Simulation.

\section{Introduction}

Formation flying technology has been extensively investigated as a means to get better performance than a single monolithic satellite. This technology guarantees a reduction in cost and has many applications such as the optical interferometry, distributed sensing, gravitational field measurements, ionospheric observation, earth observation, and 3D mapping for planetary explorers. One of the central problems of formation flying is the proper description of the relative dynamics between satellites, which is the basis of formation design and control. Conventionally, linearized equations of relative motion such as Hill-Clohessy-Wiltshire (HCW) equations (Hill 1878; Clohessy and Wiltshire 1960), which are linearized about a reference orbit or the formation center, have been used due to their simplicity. In the presence of perturbations, however, the formation configuration based on linearized equations would be destroyed. Therefore, control forces must be applied to maintain the desired configuration for a specific mission. This formation-keeping problem for multiple satellites has been widely investigated by various researchers. Yan et al. (2000) designed a pulse-based periodic controller to keep a formation using the $\mathrm{HCW}$ equations and the linear quadratic regulation technique. The main drawback to this paper is that the earth oblateness gravity perturbation is neglected, hence, Sparks (2000) proposed a feedback control strategy in the presence of this perturbation. $\mathrm{He}$ designed a controller using discrete time linear quadratic regula-

${ }^{1}$ Graduate Student, Dept. of Aerospace and Mechanical Engineering, Univ. of Southern California, Los Angeles, CA 90089-1453 (corresponding author). E-mail: hancheoc@usc.edu

${ }^{2}$ Member, Engineering Staff, F-35 JSF Program Section, Northrop Grumman Co., 1840 Century Park East, Los Angeles, CA 90067-2199. E-mail: adam.yu@ngc.com

Note. This manuscript was submitted on March 26, 2009; approved on April 29, 2009; published online on May 4, 2009. Discussion period open until March 1, 2010; separate discussions must be submitted for individual papers. This paper is part of the Journal of Aerospace Engineering, Vol. 22, No. 4, October 1, 2009. CASCE, ISSN 0893-1321/ 2009/4-445-455/\$25.00. tor laws based on the HCW equations. Based on the HCW equations, Sabol et al. (2001) investigated several satellite formation flying designs and their evolution through time. Using the variation of orbital elements, they designed formation-keeping schemes in the presence of various perturbations. Armellin et al. (2004) developed a real-time control strategy that includes both reconfiguration and formation keeping. They solved these two problems by generating a control sequence based on a discretization of the differential constraints and a parametrization of the controls. Qingsong et al. (2004) solved this formation-keeping problem by using a low-thrust fuzzy control method. However, Yan and others (Yan et al. 2000; Sparks 2000; Sabol et al. 2001; Armellin et al. 2004; Qingsong et al. 2004) made use of the linearized equations of relative motion, and hence the methods thus derived are suitable only for very close formations.

Although, as pointed out earlier, there has been an extensive literature regarding formation flying to date, the effects that result from the nonlinearities remain yet to be fully modeled. Karlgaard and Lutze (2003) approached the problem using spherical coordinates and perturbation techniques to extend the HCW equations that are correct to second order. Richardson and Mitchell (2003) obtained the solution that includes the third-order nonlinearities by enforcing periodic motion. Kasdin et al. (2005) used a Hamiltonian formulation to solve the problem to include second-order nonlinearity effects, but with $J_{2}$ perturbations.

In this paper, assuming unbounded and time-varying lowthrust burns throughout the maneuver, the formation-keeping problem is exactly and explicitly solved without any restriction about the distance between satellites. Also, this paper derives the exact control force to maintain the configuration of the formation. We use a new approach for constrained dynamic systems proposed by Udwadia and Kalaba (Udwadia 2000, 2002, 2003, 2005, 2008; Kalaba and Udwadia 1993; Udwadia and Kalaba 1992, 2002), which is based on Gauss's principle. The Udwadia-Kalaba equation unlike the Lagrange's equation handles both holonomic and nonholonomic constraints with equal ease. This equation contains the generalized Moore-Penrose inverse (Udwadia and Kalaba 1999) and has been applied to highly constrained prob- 
lems in various fields of study such as robotics (Peters et al. 2005) and astrodynamics (Schutte and Dooley 2005). The major contribution of this paper is the ability to explicitly solve the complete nonlinear dynamics and control problem as a whole without the use of any approximations. In previous research, the linearized equations of relative motion described in a local moving frame or their approximate versions (that are valid only to second or third order) have been used to solve the formation-keeping problem, so it has been very challenging to obtain an exact solution.

We consider a satellite called the "chief" orbiting a central body, and require that a "deputy" satellite maintain a given required formation relative to this chief. We employ herein the twobody equation of motion described in an inertial frame of reference, and then incorporate the station-keeping requirements as constraints to obtain a simple equation of motion that completely captures all the nonlinearities. This equation exactly yields the given constrained motion and also gives an exact control force (as an explicit function of the state and time) required to maintain the formation. The results obtained can apply to the case even when the distance between satellites is so large that the linearized relative equations of motion no longer hold. The dynamics once described in the inertial frame are then recast, for convenience, into a local moving frame by the use of a transformation matrix. As a practical constraint we choose the relative configuration that is circular when projected on the local horizontal plane, which is generally called the projected circular orbit (PCO) (Vaddi et al. 2003). This projected circular formation is based on the solutions to the linearized equations, and hence the nonlinear behavior of relative motion will break down this configuration. To avoid this break down of configuration, the need to use a control force arises, which will be solved for in detail. Also, this new approach gives a general methodology that can be easily applied to any type of relative configuration.

The analysis in this paper is summarized as follows: in the section Udwadia-Kalaba equation, we briefly discuss the fundamental equation of motion for a constrained system. Next, in the section Unconstrained motion, the acceleration for the unconstrained motion is depicted, which also serves as a solution for the relative motion in the two-body problem. In the section Constrained motion, the constraint equation and the exact control force are derived in the inertial frame which forces the deputy to maintain the required PCO configuration. Finally, in the section Numerical simulations, numerical simulations are presented to demonstrate the brevity and the accuracy of the method.

\section{Udwadia-Kalaba Equation}

This section deals with the fundamental equation for a constrained system, which we will call the Udwadia-Kalaba equation. Consider a point-mass satellite in the inertial Cartesian coordinate frame. When the initial position and velocity of the satellite are known, the vectors of displacement and velocity are denoted by the following:

$$
\mathbf{x}=\left[x_{1}, x_{2}, x_{3}\right]^{T} ; \quad \dot{\mathbf{x}}=\left[\dot{x}_{1}, \dot{x}_{2}, \dot{x}_{3}\right]^{T}
$$

The forces impressed on the satellite are denoted by a 3 by 1 vector, and denoted by

$$
\mathbf{F}(t)=\left[F_{1}(t), F_{2}(t), F_{3}(t)\right]^{T}
$$

The unconstrained motion of the system can be expressed as a 3 by 1 matrix equation

$$
\mathbf{M} \ddot{\mathbf{x}}=\mathbf{F}[\mathbf{x}(t), \dot{\mathbf{x}}(t), t]
$$

or

$$
\ddot{\mathbf{x}}=\mathbf{M}^{-1} \mathbf{F}[\mathbf{x}(t), \dot{\mathbf{x}}(t), t]=\mathbf{a}(t)
$$

where $\mathbf{M}=m \mathbf{I}_{\mathbf{3} \times \mathbf{3}}=$ diagonal mass matrix; $m=$ mass of the satellite; $\mathbf{I}_{\mathbf{3} \times \mathbf{3}}=3$ by 3 identity matrix; and $\mathbf{a}(t)=$ acceleration at the time $t$ of the unconstrained system. Furthermore, the system is constrained through the application of the following set of consistent constraint equations:

$$
\mathbf{A}(\mathbf{x}(t), \dot{\mathbf{x}}(t), t) \ddot{\mathbf{x}}=\mathbf{b}(\mathbf{x}(t), \dot{\mathbf{x}}(t), t)
$$

where the matrix $\mathbf{A}=m$ by 3 matrix and $\mathbf{b}=m$ by 1 vector, where $m$ is the number of constraints. The application of constraints on the unconstrained system causes additional forces to be applied to the unconstrained system and the equation of motion of the constrained system becomes

$$
\mathbf{M} \ddot{\mathbf{x}}(t)=\mathbf{F}(\mathbf{x}(t), \dot{\mathbf{x}}(t), t)+\mathbf{F}^{\mathbf{c}}
$$

where $\mathbf{F}^{\mathbf{c}}$ =additional constraint force vector that arises due to the application of the constraints. Udwadia and Kalaba proposed the following equation of motion for the constrained system (uniquely described at each instant of time) (Udwadia and Kalaba 2008):

$$
\mathbf{M} \ddot{\mathbf{x}}=\mathbf{F}+\mathbf{M}^{1 / 2}\left(\mathbf{A M}^{-1 / 2}\right)^{+}(\mathbf{b}-\mathbf{A a})
$$

where the superscript " + " represents the generalized MoorePenrose inverse. In this paper, Eq. (7) is referred to as the Udwadia-Kalaba equation. It is important to note that the Udwadia-Kalaba equation should be described in the inertial frame of reference. Then, the constraint force vector $\mathbf{F}^{\mathbf{c}}(t)$ is described as

$$
\mathbf{F}^{\mathbf{c}}(t)=\mathbf{M}^{1 / 2}\left(\mathbf{A} \mathbf{M}^{-1 / 2}\right)^{+}(\mathbf{b}-\mathbf{A a})
$$

Since the generalized Moore-Penrose inverse of a matrix is unique, the control force is uniquely and explicitly calculated by Eq. (8), regardless of whether the constraints given by Eq. (5) are holonomic or nonholonomic. It is straightforward to show that the Udwadia-Kalaba equation satisfies the constraint Eq. (5). Using the fact that $\mathbf{M}=m \mathbf{I}_{\mathbf{3} \times \mathbf{3}}$ in this paper, as we are concerned with only a single deputy satellite, the Udwadia-Kalaba equation can be further simplified to (Udwadia and Kalaba 2008)

$$
\ddot{\mathbf{x}}=\mathbf{a}+\mathbf{A}^{+}(\mathbf{b}-\mathbf{A} \mathbf{a})
$$

$$
\mathbf{M} \ddot{\mathbf{x}}=\mathbf{F}+\mathbf{M A}^{+}(\mathbf{b}-\mathbf{A a})
$$

\section{Unconstrained Motion}

The unconstrained motion represents the relative motion without the application of a control force on the system of satellites. The chief and the deputy satellites move only under the influence of gravity, and hence we consider "unconstrained" motion and "uncontrolled" motion to have the same meaning. In the case of constrained motion, proper control forces are needed to be applied on the unconstrained system to meet the constraint requirements. Hence, we consider "constrained" motion and "controlled" motion to also have the same meaning.

When a point-mass satellite is orbiting around the Earth, the unconstrained acceleration of the system is expressed in the inertial coordinate system as follows (Prussing and Conway 1993): 


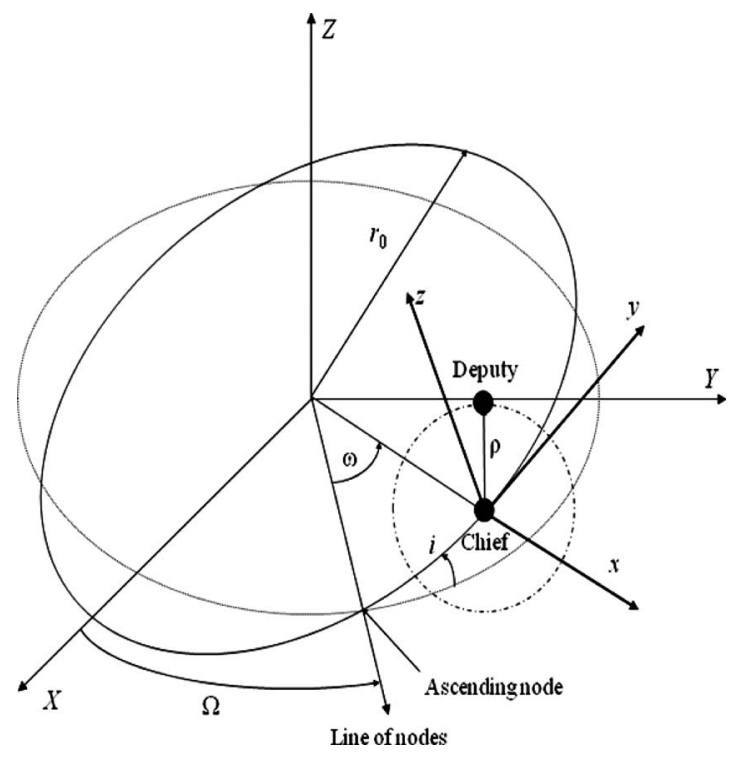

Fig. 1. ECI frame $(X-Y-Z)$ and Hill frame $(x-y-z)$. The distance between the chief and the deputy must be maintained to be $\rho$ in the $y z$-plane of the local, rotating coordinate frame $x-y-z$.

$$
\mathbf{a}=-\frac{G M}{\left(X^{2}+Y^{2}+Z^{2}\right)^{3 / 2}}\left[\begin{array}{l}
X \\
Y \\
Z
\end{array}\right]
$$

where $G=$ universal gravitational constant and $M=$ mass of a central body, the Earth in this paper. Eq. (11) is described in the inertial frame, more precisely the Earth-centered inertial (ECI) frame. This coordinate frame originates at the center of the Earth, the $X$ axis points toward the vernal equinox, the $Z$ axis extends through the North Pole, and the $Y$ axis completes the right-handed rule (Fig. 1).

\section{Constrained Motion}

Assuming the chief satellite is in a circular orbit with a fixed inclination under no perturbation, our goal is to maintain the distance between the chief and deputy to be constant, say $\rho$, when projected on the local horizontal plane. Also, to prevent the $x$ coordinate from diverging, another constraint between $x$ and $z$ coordinates is applied. To describe these constraints, it is convenient to define a new coordinate frame, called the Hill frame or the local-vertical and local-horizontal frame. The origin of this frame is located at the chief, the $x$ axis lies in the radial direction, the $y$ axis is in the along-track direction, and the $z$ axis lies along the orbital angular momentum vector, thus completing a righthanded coordinate system (see Fig. 1). In this frame, the constraint equations can be written as

$$
\begin{gathered}
y^{2}+z^{2}=\rho^{2} \\
2 x-z=0
\end{gathered}
$$

where $\rho=$ constant distance between the chief and the deputy. Eq. $(12 b)$ is added to make the relative motion to be bounded in every axis, and it also matches with the solutions of the HCW equations satisfying the constraint Eq. (12a) (Sabol et al. 2001). Since Eqs. $(12 a)$ and $(12 b)$ are holonomic constraints, we differentiate Eqs. (12a) and (12b) twice with respect to time and the equations become

$$
\begin{gathered}
y \ddot{y}+z \ddot{z}=-\dot{y}^{2}-\dot{z}^{2} \\
2 \ddot{x}-\ddot{z}=0
\end{gathered}
$$

where $x, y$, and $z=$ described in the Hill frame. Since the Hill frame is a rotating or noninertial frame, we must transform Eq. (13) into the one described in the ECI (inertial) frame.

The transformation can be accomplished as follows: first, we rotate by $\Omega$ about the $Z$ axis of the ECI frame where $\Omega$ is the longitude of the ascending node of the chief, the angle between the $X$ axis and the line of node. We will denote this rotation matrix by $\mathbf{R}_{3}(\Omega)$ where the subscript 3 means the rotation about the third axis, $Z$ axis in this case

$$
\mathbf{R}_{\mathbf{3}}(\Omega)=\left[\begin{array}{ccc}
\cos (\Omega) & \sin (\Omega) & 0 \\
-\sin (\Omega) & \cos (\Omega) & 0 \\
0 & 0 & 1
\end{array}\right]
$$

Next, we rotate the system by $i$ about the first axis, where $i$ is the inclination of the chief, the angle between the equatorial plane and the chief's orbital plane. We denote this rotation matrix as $\mathbf{R}_{\mathbf{1}}(i)$

$$
\mathbf{R}_{\mathbf{1}}(i)=\left[\begin{array}{ccc}
1 & 0 & 0 \\
0 & \cos (i) & \sin (i) \\
0 & -\sin (i) & \cos (i)
\end{array}\right]
$$

It is important to note that both $\Omega$ and $i$ are constant because the chief is in a circular orbit under no perturbation, so both $\mathbf{R}_{\mathbf{3}}(\Omega)$ and $\mathbf{R}_{\mathbf{1}}(i)$ are constant. Then, we rotate the system by $\omega$ about the third axis, where $\omega$ is the argument of latitude of the chief, the angle measured between the ascending node and the chief's position vector. We denote this rotation matrix as $\mathbf{R}_{\mathbf{3}}(\omega)$

$$
\mathbf{R}_{\mathbf{3}}(\omega)=\left[\begin{array}{ccc}
\cos (\omega) & \sin (\omega) & 0 \\
-\sin (\omega) & \cos (\omega) & 0 \\
0 & 0 & 1
\end{array}\right]
$$

Note that $\omega$ is not constant because the chief is moving. $\omega$ can be represented as

$$
\omega=\omega_{0}+n\left(t-t_{0}\right)
$$

where $\omega_{0}=$ argument of latitude at the initial time, $t_{0}$, and $n$ denotes the constant mean motion of the chief

$$
n=\sqrt{\frac{G M}{r_{0}^{3}}}
$$

Here, $r_{0}=$ radius of the chief's circular orbit. Finally, we translate the system by $-r_{0}$ along the $x$ axis to coincide the origin with the chief's position. Then, the final transformation equation which maps the ECI frame $(X, Y, Z)$ to the Hill frame $(x, y, z)$ becomes

$$
\left[\begin{array}{c}
x+r_{0} \\
y \\
z
\end{array}\right]=\mathbf{R}_{\mathbf{3}}(\omega) \mathbf{R}_{\mathbf{1}}(i) \mathbf{R}_{\mathbf{3}}(\Omega)\left[\begin{array}{c}
X \\
Y \\
Z
\end{array}\right]
$$

Let us define the total rotation matrix as $\mathbf{R}$

$$
\mathbf{R} \equiv \mathbf{R}_{\mathbf{3}}(\omega) \mathbf{R}_{\mathbf{1}}(i) \mathbf{R}_{\mathbf{3}}(\Omega)
$$

Using Eqs. (14)-(16), we get 


$$
\mathbf{R}=\left[\begin{array}{ccc}
\cos (\Omega) \cos (\omega)-\sin (\Omega) \cos (i) \sin (\omega) & \sin (\Omega) \cos (\omega)+\cos (\Omega) \cos (i) \sin (\omega) & \sin (i) \sin (\omega) \\
-\cos (\Omega) \sin (\omega)-\sin (\Omega) \cos (i) \cos (\omega) & \cos (\Omega) \cos (i) \cos (\omega)-\sin (\Omega) \sin (\omega) & \sin (i) \cos (\omega) \\
\sin (\Omega) \sin (i) & -\cos (\Omega) \sin (i) & \cos (i)
\end{array}\right]
$$

The first constraint equation [Eq. (13a)] contains only $y$ and $z$, so we use only the last two rows of the matrix $\mathbf{R}$ and define the following matrix:

$$
\overline{\mathbf{R}} \equiv\left[\begin{array}{ccc}
-\cos (\Omega) \sin (\omega)-\sin (\Omega) \cos (i) \cos (\omega) & \cos (\Omega) \cos (i) \cos (\omega)-\sin (\Omega) \sin (\omega) & \sin (i) \cos (\omega) \\
\sin (\Omega) \sin (i) & -\cos (\Omega) \sin (i) & \cos (i)
\end{array}\right]
$$

Differentiating the matrix $\overline{\mathbf{R}}$ once and twice with respect to time, we get the following:

$$
\begin{gathered}
\dot{\overline{\mathbf{R}}}=\left[\begin{array}{ccc}
-n \cos (\Omega) \cos (\omega)+n \sin (\Omega) \cos (i) \sin (\omega) & -n \cos (\Omega) \cos (i) \sin (\omega)-n \sin (\Omega) \cos (\omega) & -n \sin (i) \sin (\omega) \\
0 & 0 & 0
\end{array}\right] \\
\ddot{\mathbf{R}}=\left[\begin{array}{ccc}
n^{2} \cos (\Omega) \sin (\omega)+n^{2} \sin (\Omega) \cos (i) \cos (\omega) & -n^{2} \cos (\Omega) \cos (i) \cos (\omega)+n^{2} \sin (\Omega) \sin (\omega) & -n^{2} \sin (i) \cos (\omega) \\
0 & 0 & 0
\end{array}\right]
\end{gathered}
$$

Also, the second constraint equation [Eq. (13b)] contains only $x$ and $z$, so we use only the first and third rows of the matrix $\mathbf{R}$ and define the following matrix:

$$
\tilde{\mathbf{R}} \equiv\left[\begin{array}{ccc}
\cos (\Omega) \cos (\omega)-\sin (\Omega) \cos (i) \sin (\omega) & \sin (\Omega) \cos (\omega)+\cos (\Omega) \cos (i) \sin (\omega) & \sin (i) \sin (\omega) \\
\sin (\Omega) \sin (i) & -\cos (\Omega) \sin (i) & \cos (i)
\end{array}\right]
$$

Differentiating the matrix $\tilde{\mathbf{R}}$ once and twice with respect to time, we get the following:

$$
\begin{array}{r}
\dot{\tilde{\mathbf{R}}}=\left[\begin{array}{ccc}
-n \cos (\Omega) \sin (\omega)-n \sin (\Omega) \cos (i) \cos (\omega) & -n \sin (\Omega) \sin (\omega)+n \cos (\Omega) \cos (i) \cos (\omega) & n \sin (i) \cos (\omega) \\
0 & 0 & 0
\end{array}\right] \\
\ddot{\tilde{\mathbf{R}}}=\left[\begin{array}{ccc}
-n^{2} \cos (\Omega) \cos (\omega)+n^{2} \sin (\Omega) \cos (i) \sin (\omega) & -n^{2} \sin (\Omega) \cos (\omega)-n^{2} \cos (\Omega) \cos (i) \sin (\omega) & -n^{2} \sin (i) \sin (\omega) \\
0 & 0 & 0
\end{array}\right]
\end{array}
$$

If the chief is in an equatorial orbit, then its inclination is zero, the line of nodes does not exist, so $\Omega$ and $\omega$ cannot be defined. In this case the $X$ axis is considered as the node, therefore $\Omega=0, i=0$, and $\omega$ is just the angle between the $X$ axis and the position of the chief. Hence, the transformation matrix $\mathbf{R}$ is simply given by

$$
\mathbf{R}=\mathbf{R}_{\mathbf{3}}(\omega)=\left[\begin{array}{ccc}
\cos (\omega) & \sin (\omega) & 0 \\
-\sin (\omega) & \cos (\omega) & 0 \\
0 & 0 & 1
\end{array}\right]
$$

Also

$$
\begin{aligned}
& \overline{\mathbf{R}}=\left[\begin{array}{ccc}
-\sin (\omega) & \cos (\omega) & 0 \\
0 & 0 & 1
\end{array}\right] ; \\
& \dot{\overline{\mathbf{R}}}=\left[\begin{array}{ccc}
-n \cos (\omega) & -n \sin (\omega) & 0 \\
0 & 0 & 0
\end{array}\right] ; \\
& \ddot{\mathbf{R}}=\left[\begin{array}{ccc}
n^{2} \sin (\omega) & -n^{2} \cos (\omega) & 0 \\
0 & 0 & 0
\end{array}\right]
\end{aligned}
$$

and

$$
\begin{aligned}
\tilde{\mathbf{R}} & =\left[\begin{array}{ccc}
\cos (\omega) & \sin (\omega) & 0 \\
0 & 0 & 1
\end{array}\right] ; \\
\dot{\tilde{\mathbf{R}}} & =\left[\begin{array}{ccc}
-n \sin (\omega) & n \cos (\omega) & 0 \\
0 & 0 & 0
\end{array}\right] ; \\
\ddot{\tilde{\mathbf{R}}} & =\left[\begin{array}{ccc}
-n^{2} \cos (\omega) & -n^{2} \sin (\omega) & 0 \\
0 & 0 & 0
\end{array}\right]
\end{aligned}
$$

Let us consider the first constraint given by Eq. (12a). Then, from Eq. (19), $y$ and $z$ components in the Hill frame are related by

$$
\left[\begin{array}{l}
y \\
z
\end{array}\right]=\overline{\mathbf{R}}\left[\begin{array}{c}
X \\
Y \\
Z
\end{array}\right]
$$

Differentiating Eq. (31) once and twice yields the following:

$$
\begin{aligned}
& {\left[\begin{array}{c}
\dot{y} \\
\dot{z}
\end{array}\right]=\dot{\mathbf{R}}\left[\begin{array}{c}
X \\
Y \\
Z
\end{array}\right]+\overline{\mathbf{R}}\left[\begin{array}{c}
\dot{X} \\
\dot{Y} \\
\dot{Z}
\end{array}\right]} \\
& {\left[\begin{array}{c}
\ddot{y} \\
\ddot{z}
\end{array}\right]=\ddot{\overline{\mathbf{R}}}\left[\begin{array}{c}
X \\
Y \\
Z
\end{array}\right]+2 \dot{\overline{\mathbf{R}}}\left[\begin{array}{c}
\dot{X} \\
\dot{Y} \\
\dot{Z}
\end{array}\right]+\overline{\mathbf{R}}\left[\begin{array}{c}
\ddot{X} \\
\ddot{Y} \\
\ddot{Z}
\end{array}\right]}
\end{aligned}
$$

Eq. (13a) can be represented in a matrix form

$$
\left[\begin{array}{ll}
y & z
\end{array}\right]\left[\begin{array}{l}
\ddot{y} \\
\ddot{z}
\end{array}\right]=-\left[\begin{array}{ll}
\dot{y} & \dot{z}
\end{array}\right]\left[\begin{array}{l}
\dot{y} \\
\dot{z}
\end{array}\right]
$$

Substituting Eqs. (31)-(33) into Eq. (34), we get the first constraint equation described in the ECI frame 


$$
\begin{gathered}
{\left[\begin{array}{lll}
X & Y & Z
\end{array}\right] \overline{\mathbf{R}}^{T}\left\{\ddot{\mathbf{R}}\left[\begin{array}{c}
X \\
Y \\
Z
\end{array}\right]+2 \dot{\overline{\mathbf{R}}}\left[\begin{array}{c}
\dot{X} \\
\dot{Y} \\
\dot{Z}
\end{array}\right]+\overline{\mathbf{R}}\left[\begin{array}{c}
\ddot{X} \\
\ddot{Y} \\
\ddot{Z}
\end{array}\right]\right\}=-\left\{\left[\begin{array}{lll}
X & Y & Z
\end{array}\right] \dot{\mathbf{R}}^{T}\right.} \\
\left.+\left[\begin{array}{lll}
\dot{X} & \dot{Y} & \dot{Z}
\end{array}\right] \overline{\mathbf{R}}^{T}\right\}\left\{\dot{\mathbf{R}}\left[\begin{array}{c}
X \\
Y \\
Z
\end{array}\right]+\overline{\mathbf{R}}\left[\begin{array}{c}
\dot{X} \\
\dot{Y} \\
\dot{Z}
\end{array}\right]\right\}
\end{gathered}
$$

Eq. (35) can be rearranged into the following:

$$
\begin{aligned}
{\left[\begin{array}{lll}
X & Y & Z
\end{array}\right] \overline{\mathbf{R}}^{T} \overline{\mathbf{R}}\left[\begin{array}{c}
\ddot{X} \\
\ddot{Y} \\
\ddot{Z}
\end{array}\right]=} & -\left[\begin{array}{lll}
X & Y & Z
\end{array}\right] \overline{\mathbf{R}}^{T} \ddot{\overline{\mathbf{R}}}\left[\begin{array}{c}
X \\
Y \\
Z
\end{array}\right] \\
& -2\left[\begin{array}{lll}
X & Y & Z
\end{array}\right] \overline{\mathbf{R}}^{T} \dot{\overline{\mathbf{R}}}\left[\begin{array}{c}
\dot{X} \\
\dot{Y} \\
\dot{Z}
\end{array}\right] \\
& -\left[\begin{array}{lll}
X & Y & Z
\end{array}\right] \dot{\overline{\mathbf{R}}}^{T} \dot{\overline{\mathbf{R}}}\left[\begin{array}{c}
X \\
Y \\
Z
\end{array}\right] \\
& -2\left[\begin{array}{lll}
X & Y & Z
\end{array}\right] \dot{\overline{\mathbf{R}}}^{T} \overline{\mathbf{R}}\left[\begin{array}{c}
\dot{X} \\
\dot{X} \\
\dot{Z} \\
\dot{Z}
\end{array}\right] \\
& -\left[\begin{array}{lll}
\dot{X} & \dot{Y} & \dot{Z}
\end{array}\right] \overline{\mathbf{R}}^{T} \overline{\mathbf{R}}\left[\begin{array}{c}
\dot{X} \\
\dot{Y} \\
\dot{Z}
\end{array}\right]
\end{aligned}
$$

Next, let us consider the second constraint Eq. (12b). Then, from Eq. (19), $x$ - and $z$-components in the Hill frame are related by

$$
\left[\begin{array}{l}
x \\
z
\end{array}\right]=\widetilde{\mathbf{R}}\left[\begin{array}{c}
X \\
Y \\
Z
\end{array}\right]
$$

Differentiating Eq. (37) once and twice yields the following:

$$
\begin{aligned}
& {\left[\begin{array}{c}
\dot{x} \\
\dot{z}
\end{array}\right]=\dot{\tilde{\mathbf{R}}}\left[\begin{array}{c}
X \\
Y \\
Z
\end{array}\right]+\widetilde{\mathbf{R}}\left[\begin{array}{c}
\dot{X} \\
\dot{Y} \\
\dot{Z}
\end{array}\right]} \\
& {\left[\begin{array}{c}
\ddot{X} \\
\ddot{Z}
\end{array}\right]=\ddot{\widetilde{\mathbf{R}}}\left[\begin{array}{c}
X \\
Y \\
Z
\end{array}\right]+2 \dot{\tilde{\mathbf{R}}}\left[\begin{array}{c}
\dot{X} \\
\dot{Y} \\
\dot{Z}
\end{array}\right]+\widetilde{\mathbf{R}}\left[\begin{array}{c}
\ddot{X} \\
\ddot{Y} \\
\ddot{Z}
\end{array}\right]}
\end{aligned}
$$

Eq. (13b) can be represented in a matrix form

$$
\left[\begin{array}{ll}
2 & -1
\end{array}\right]\left[\begin{array}{l}
\ddot{x} \\
\ddot{z}
\end{array}\right]=0
$$

Inserting Eq. (39) into Eq. (40) yields the second constraint equation described in the ECI frame

$$
\left[\begin{array}{ll}
2 & -1
\end{array}\right] \widetilde{\mathbf{R}}\left[\begin{array}{c}
\ddot{X} \\
\ddot{Y} \\
\ddot{Z}
\end{array}\right]=-\left[\begin{array}{ll}
2 & -1
\end{array}\right] \ddot{\tilde{\mathbf{R}}}\left[\begin{array}{c}
X \\
Y \\
Z
\end{array}\right]-\left[\begin{array}{ll}
4 & -2
\end{array}\right] \dot{\tilde{\mathbf{R}}}\left[\begin{array}{c}
\dot{X} \\
\dot{Y} \\
\dot{Z}
\end{array}\right]
$$

The two constraint equations [Eqs. (36) and (41)] can be represented in a form of Eq. (5)

$$
\mathbf{A}(\mathbf{x}(t), \dot{\mathbf{x}}(t), t) \ddot{\mathbf{x}}=\mathbf{b}(\mathbf{x}(t), \dot{\mathbf{x}}(t), t)
$$

More specifically, we obtain the following vector equation:

$$
\left[\begin{array}{lll}
A_{11} & A_{12} & A_{13} \\
A_{21} & A_{22} & A_{23}
\end{array}\right]\left[\begin{array}{l}
\ddot{X} \\
\ddot{Y} \\
\ddot{Z}
\end{array}\right]=\left[\begin{array}{l}
b_{1} \\
b_{2}
\end{array}\right]
$$

Then, each component of the matrices $\mathbf{A}$ and $\mathbf{b}$ can be written as follows:

$$
\left[\begin{array}{lll}
A_{11} & A_{12} & A_{13}
\end{array}\right]=\left[\begin{array}{lll}
X & Y & Z
\end{array}\right] \overline{\mathbf{R}}^{T} \overline{\mathbf{R}}
$$

so that

$$
\begin{aligned}
A_{11} \equiv & X\left\{[\cos (\Omega) \sin (\omega)+\sin (\Omega) \cos (i) \cos (\omega)]^{2}+\sin ^{2}(\Omega) \sin ^{2}(i)\right\} \\
+ & Y\{[\cos (\Omega) \sin (\omega)+\sin (\Omega) \cos (i) \cos (\omega)][\sin (\Omega) \sin (\omega) \\
- & \left.\cos (\Omega) \cos (i) \cos (\omega)]-\sin (\Omega) \cos (\Omega) \sin ^{2}(i)\right\} \\
+ & Z\{\sin (\Omega) \sin (i) \cos (i)-\sin (i) \cos (\omega)[\cos (\Omega) \sin (\omega) \\
+ & \sin (\Omega) \cos (i) \cos (\omega)]\} \\
A_{12} \equiv & X\{[\cos (\Omega) \sin (\omega)+\sin (\Omega) \cos (i) \cos (\omega)][\sin (\Omega) \sin (\omega) \\
& \left.-\cos (\Omega) \cos (i) \cos (\omega)]-\sin (\Omega) \cos (\Omega) \sin ^{2}(i)\right\} \\
& +Y\left\{[\cos (\Omega) \cos (i) \cos (\omega)-\sin (\Omega) \sin (\omega)]^{2}\right. \\
& \left.+\cos { }^{2}(\Omega) \sin { }^{2}(i)\right\}+Z\{\sin (i) \cos (\omega)[\cos (\Omega) \cos (i) \cos (\omega) \\
& -\sin (\Omega) \sin (\omega)]-\cos (\Omega) \sin (i) \cos (i)\} \\
A_{13} \equiv & X\{\sin (\Omega) \sin (i) \cos (i)-\sin (i) \cos (\omega)[\cos (\Omega) \sin (\omega) \\
& +\sin (\Omega) \cos (i) \cos (\omega)]\}+Y\{\sin (i) \cos (\omega) \\
& \times[\cos (\Omega) \cos (i) \cos (\omega)-\sin (\Omega) \sin (\omega)] \\
& -\cos (\Omega) \sin (i) \cos (i)\}+Z\left[\sin { }^{2}(i) \cos (\omega)+\cos ^{2}(i)\right]
\end{aligned}
$$

and

$$
\left[\begin{array}{lll}
A_{21} & A_{22} & A_{23}
\end{array}\right]=\left[\begin{array}{ll}
2 & -1
\end{array}\right] \tilde{\mathbf{R}}
$$

so that

$$
A_{21}=2 \cos (\Omega) \cos (\omega)-2 \sin (\Omega) \cos (i) \sin (\omega)-\sin (\Omega) \sin (i)
$$

$$
A_{22}=2 \sin (\Omega) \cos (\omega)+2 \cos (\Omega) \cos (i) \sin (\omega)+\cos (\Omega) \sin (i)
$$

$$
A_{23}=2 \sin (i) \sin (\omega)-\cos (i)
$$


In addition

$$
\begin{aligned}
b_{1}= & -\left[\begin{array}{lll}
X & Y & Z
\end{array}\right] \overline{\mathbf{R}}^{T} \ddot{\mathbf{R}}\left[\begin{array}{c}
X \\
Y \\
Z
\end{array}\right]-2\left[\begin{array}{lll}
X & Y & Z
\end{array}\right] \overline{\mathbf{R}}^{T} \dot{\overline{\mathbf{R}}}\left[\begin{array}{c}
\dot{X} \\
\dot{Y} \\
\dot{Z}
\end{array}\right] \\
& -\left[\begin{array}{lll}
X & Y & Z
\end{array}\right] \dot{\overline{\mathbf{R}}}^{T} \dot{\overline{\mathbf{R}}}\left[\begin{array}{c}
X \\
Y \\
Z
\end{array}\right]-2\left[\begin{array}{lll}
X & Y & Z
\end{array}\right] \dot{\overline{\mathbf{R}}}^{T} \overline{\mathbf{R}}\left[\begin{array}{c}
\dot{X} \\
\dot{Y} \\
\dot{Z}
\end{array}\right] \\
& -\left[\begin{array}{lll}
\dot{X} & \dot{Y} & \dot{Z}
\end{array}\right] \overline{\mathbf{R}}^{T} \overline{\mathbf{R}}\left[\begin{array}{c}
\dot{X} \\
\dot{Y} \\
\dot{Z}
\end{array}\right]
\end{aligned}
$$

and

$$
b_{2}=-\left[\begin{array}{ll}
2 & -1
\end{array}\right] \ddot{\tilde{\mathbf{R}}}\left[\begin{array}{c}
X \\
Y \\
Z
\end{array}\right]-\left[\begin{array}{ll}
4 & -2
\end{array}\right] \dot{\tilde{\mathbf{R}}}\left[\begin{array}{c}
\dot{X} \\
\dot{Y} \\
\dot{Z}
\end{array}\right]
$$

The generalized Moore-Penrose inverse of the matrix A can be obtained in a following closed form (Udwadia and Kalaba 2008):

$$
\mathbf{A}^{+}=\left[\left(\mathbf{A}_{1}^{+}-\beta \mathbf{c}^{+}\right) \mid \mathbf{c}^{+}\right]
$$

where

$$
\begin{gathered}
\mathbf{A}_{1}^{+}=\frac{1}{A_{11}^{2}+A_{12}^{2}+A_{13}^{2}}\left[\begin{array}{l}
A_{11} \\
A_{12} \\
A_{13}
\end{array}\right] \\
\beta=\frac{A_{11} A_{21}+A_{12} A_{22}+A_{13} A_{23}}{A_{11}^{2}+A_{12}^{2}+A_{13}^{2}} \\
\mathbf{c}^{+}=\frac{1}{A_{21}^{2}+A_{22}^{2}+A_{23}^{2}-\beta^{2}\left(A_{11}^{2}+A_{12}^{2}+A_{13}^{2}\right)}\left(\left[\begin{array}{l}
A_{21} \\
A_{22} \\
A_{23}
\end{array}\right]-\beta\left[\begin{array}{l}
A_{11} \\
A_{12} \\
A_{13}
\end{array}\right]\right)
\end{gathered}
$$

Finally, substituting all these terms in the Udwadia-Kalaba equation [Eq. (9)]

$$
\begin{aligned}
\ddot{\mathbf{x}}= & \mathbf{a}+\mathbf{A}^{+}(\mathbf{b}-\mathbf{A a})=-\frac{G M}{\left(X^{2}+Y^{2}+Z^{2}\right)^{3 / 2}}\left[\begin{array}{c}
X \\
Y \\
Z
\end{array}\right]+\left[\left(\mathbf{A}_{1}^{+}-\beta \mathbf{c}^{+}\right) \mid \mathbf{c}^{+}\right] \\
& \times\left[\begin{array}{l}
b_{1} \\
b_{2}
\end{array}\right]+\frac{G M}{\left(X^{2}+Y^{2}+Z^{2}\right)^{3 / 2}}\left[\left(\mathbf{A}_{1}^{+}-\beta \mathbf{c}^{+}\right) \mid \mathbf{c}^{+}\right]\left[\begin{array}{lll}
A_{11} & A_{12} & A_{13} \\
A_{21} & A_{22} & A_{23}
\end{array}\right] \\
& \times\left[\begin{array}{c}
X \\
Y \\
Z
\end{array}\right]
\end{aligned}
$$

The constraint force that is needed to satisfy our constraints can be explicitly obtained

$$
\begin{aligned}
\mathbf{F}^{\mathbf{c}}= & \mathbf{M} \mathbf{A}^{+}(\mathbf{b}-\mathbf{A} \mathbf{a})=m\left[\left(\mathbf{A}_{1}^{+}-\beta \mathbf{c}^{+}\right) \mid \mathbf{c}^{+}\right]\left[\begin{array}{l}
b_{1} \\
b_{2}
\end{array}\right] \\
& +\frac{G M m}{\left(X^{2}+Y^{2}+Z^{2}\right)^{3 / 2}}\left[\left(\mathbf{A}_{1}^{+}-\beta \mathbf{c}^{+}\right) \mid \mathbf{c}^{+}\right]\left[\begin{array}{lll}
A_{11} & A_{12} & A_{13} \\
A_{21} & A_{22} & A_{23}
\end{array}\right]\left[\begin{array}{l}
X \\
Y \\
Z
\end{array}\right]
\end{aligned}
$$

where $m=$ mass of the deputy satellite. In Eqs. (53) and (54), $\mathbf{A}_{1}^{+}$, $\beta$, and $\mathbf{c}^{+}$are explicitly given in Eqs. (50)-(52) as a closed form.

\section{Numerical Simulations}

In this section, the analytical results in the previous section are verified by numerical simulations. The radius of the chief's orbit is assumed to be $7.0 \times 10^{6} \mathrm{~m}$ and the mean motion $n$ is 0.001078 $\mathrm{rad} / \mathrm{s}$. The deputy is desired to be maintained the PCO with $\rho$ $=50 \mathrm{~km}$. Since the value of $\rho$ is not small, using the HCW equations is not a good idea to solve this problem. $\Omega, i$, and $\omega_{0}$ of the chief are given as $30^{\circ}, 80^{\circ}$, and $0^{\circ}$, respectively. Therefore the initial condition for the chief in the ECI frame is given by

$\begin{aligned} \mathbf{X}_{0}^{*} & =\left[\begin{array}{c}r_{0} \cos (\Omega) \\ r_{0} \sin (\Omega) \\ 0\end{array}\right]=\left[\begin{array}{c}6.062 \times 10^{6} \mathrm{~m} \\ 3.5 \times 10^{6} \mathrm{~m} \\ 0 \mathrm{~m}\end{array}\right] ; \\ \dot{\mathbf{X}}_{\mathbf{0}}^{*} & =\left[\begin{array}{c}-v_{0} \cos (i) \sin (\Omega) \\ v_{0} \cos (i) \cos (\Omega) \\ v_{0} \sin (i)\end{array}\right]=\left[\begin{array}{c}-6.553 \times 10^{2} \mathrm{~m} / \mathrm{s} \\ 1.135 \times 10^{3} \mathrm{~m} / \mathrm{s} \\ 7.432 \times 10^{3} \mathrm{~m} / \mathrm{s}\end{array}\right]\end{aligned}$

where the subscript 0 means the values at the initial time, the superscript ${ }^{*}$ denotes the vector associated with the chief satellite and $v_{0}$ is the constant orbital speed of the chief, which is determined by the vis-viva equation (Prussing and Conway 1993)

$$
v_{0}=\sqrt{\frac{G M}{r_{0}}}=7.547 \times 10^{3} \mathrm{~m} / \mathrm{s}
$$

Next, we must be careful when dealing with the initial condition of the deputy, because the initial condition must also satisfy the constraint equations. In this paper, the constraints are holonomic, so both Eqs. (12) and (13) must hold. Eqs. (12) and (13) can be represented in the ECI frame as

$$
\begin{gathered}
{\left[\begin{array}{lll}
X & Y & Z
\end{array}\right] \overline{\mathbf{R}}^{T} \overline{\mathbf{R}}\left[\begin{array}{c}
X \\
Y \\
Z
\end{array}\right]-\rho^{2}=0,\left[\begin{array}{ll}
2 & -1
\end{array}\right] \tilde{\mathbf{R}}\left[\begin{array}{c}
X \\
Y \\
Z
\end{array}\right]=0} \\
{\left[\begin{array}{lll}
X & Y & Z
\end{array}\right] \overline{\mathbf{R}}^{T} \dot{\overline{\mathbf{R}}}\left[\begin{array}{c}
X \\
Y \\
Z
\end{array}\right]+\left[\begin{array}{lll}
X & Y & Z
\end{array}\right] \overline{\mathbf{R}}^{T} \overline{\mathbf{R}}\left[\begin{array}{c}
\dot{X} \\
\dot{Y} \\
\dot{Z}
\end{array}\right]=0,\left[\begin{array}{ll}
2 & -1
\end{array}\right] \dot{\mathbf{R}}\left[\begin{array}{c}
X \\
Y \\
Z
\end{array}\right]} \\
+\left[\begin{array}{ll}
2 & -1
\end{array}\right] \tilde{\mathbf{R}}\left[\begin{array}{c}
\dot{Y} \\
\dot{X} \\
\dot{Z}
\end{array}\right]=0
\end{gathered}
$$

Then, the initial condition for the deputy satellite must satisfy the 
following equations:

$$
\begin{gathered}
{\left[\begin{array}{lll}
X_{0} & Y_{0} & Z_{0}
\end{array}\right] \overline{\mathbf{R}}_{0}^{T} \overline{\mathbf{R}}_{0}\left[\begin{array}{c}
X_{0} \\
Y_{0} \\
Z_{0}
\end{array}\right]-\rho^{2}=0,\left[\begin{array}{ll}
2 & -1
\end{array}\right] \widetilde{\mathbf{R}}_{\mathbf{0}}\left[\begin{array}{c}
X_{0} \\
Y_{0} \\
Z_{0}
\end{array}\right]=0} \\
{\left[\begin{array}{lll}
X_{0} & Y_{0} & Z_{0}
\end{array}\right] \overline{\mathbf{R}}_{0}^{T} \dot{\mathbf{R}}_{0}\left[\begin{array}{c}
X_{0} \\
Y_{0} \\
Z_{0}
\end{array}\right]+\left[\begin{array}{lll}
X_{0} & Y_{0} & Z_{0}
\end{array}\right] \overline{\mathbf{R}}_{\mathbf{0}}^{T} \overline{\mathbf{R}}_{\mathbf{0}}\left[\begin{array}{c}
\dot{X}_{0} \\
\dot{Y}_{0} \\
\dot{Z}_{0}
\end{array}\right]} \\
=0,\left[\begin{array}{ll}
2 & -1
\end{array}\right] \dot{\tilde{\mathbf{R}}}_{\mathbf{0}}\left[\begin{array}{c}
X_{0} \\
Y_{0} \\
Z_{0}
\end{array}\right]+\left[\begin{array}{ll}
2 & -1
\end{array}\right] \widetilde{\mathbf{R}}_{\mathbf{0}}\left[\begin{array}{c}
\dot{X}_{0} \\
\dot{Y}_{0} \\
\dot{Z}_{0}
\end{array}\right]=0
\end{gathered}
$$

For practical use, the general initial conditions that satisfy the constraint equations [Eqs. (59) and (60)] are proposed by Vaddi et al. (2003)

$$
\begin{gathered}
x_{0}=\frac{\rho}{2} \sin \alpha_{0} ; \quad y_{0}=\rho \cos \alpha_{0} ; \quad z_{0}=\rho \sin \alpha_{0} \\
\dot{x}_{0}=\frac{\rho}{2} n \cos \alpha_{0} ; \quad \dot{y}_{0}=-\rho n \sin \alpha_{0} ; \quad \dot{z}_{0}=\rho n \cos \alpha_{0}
\end{gathered}
$$

where $\rho=50 \mathrm{~km} ; \alpha_{0}=$ phase angle between deputy satellites; and the mean motion $n$ is $0.001078 \mathrm{rad} / \mathrm{s}$ as before. If we use $\alpha_{0}$ $=0.55 \mathrm{rad}$, Eq. (61) will yield

$$
\begin{gathered}
x_{0}=1.3067 \times 10^{4} \mathrm{~m} ; \quad y_{0}=4.2626 \times 10^{4} \mathrm{~m} ; \\
z_{0}=2.6134 \times 10^{4} \mathrm{~m} \\
\dot{x}_{0}=22.9784 \mathrm{~m} / \mathrm{s} ; \quad \dot{y}_{0}=-28.1764 \mathrm{~m} / \mathrm{s} ; \dot{z}_{0}=45.9568 \mathrm{~m} / \mathrm{s}
\end{gathered}
$$

The initial conditions given by Eqs. (61) and (62) are designed primarily to keep the PCO without any application of control forces by assuming that the linearized HCW equations are valid, and so these initial conditions under this assumption lead to a circular orbit. However, we shall show that when the full nonlinear system is considered, the orbit no longer remains circular, and in what follows we will determine the explicit control force needed to get a circular orbit.

Eqs. (61) and (62) are described in the Hill frame. To use the Udwadia-Kalaba equation, we need to transform these values into the ones in ECI frame by using Eq. (19)

$$
\begin{aligned}
& \mathbf{X}_{\mathbf{0}}=\left[\begin{array}{c}
X_{0} \\
Y_{0} \\
Z_{0}
\end{array}\right]=\left[\begin{array}{c}
6.0827 \times 10^{6} \mathrm{~m} \\
3.4907 \times 10^{6} \mathrm{~m} \\
4.6517 \times 10^{6} \mathrm{~m}
\end{array}\right] ; \\
& \dot{\mathbf{X}}_{\mathbf{0}}=\left[\begin{array}{c}
\dot{X}_{0} \\
\dot{Y}_{0} \\
\dot{Z}_{0}
\end{array}\right]=\left[\begin{array}{c}
-651.3041 \mathrm{~m} / \mathrm{s} \\
1,082.1 \mathrm{~m} / \mathrm{s} \\
7,426.4 \mathrm{~m} / \mathrm{s}
\end{array}\right]
\end{aligned}
$$

Also, we set three times the orbital period of the chief as the simulation time in which the period is given by

$$
P=\frac{2 \pi}{n}=5.828 \times 10^{3} \mathrm{~s}=1.619 \mathrm{~h}
$$

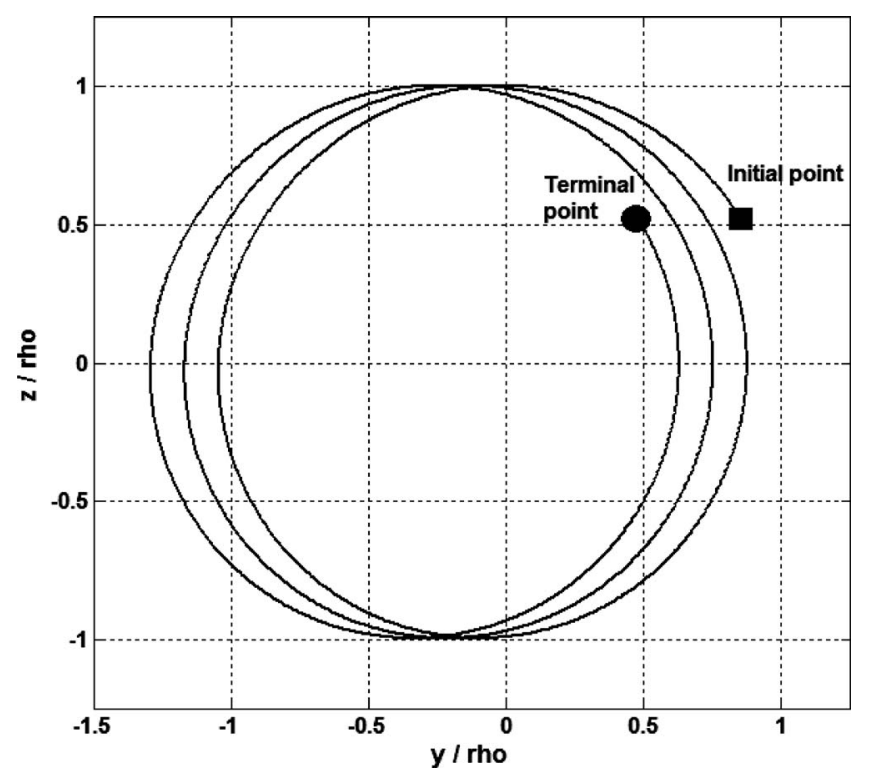

Fig. 2. Unconstrained motion in the $y z$-plane

First, we simulate the unconstrained motion. The ODE solver ODE45 in MATLAB was used to numerically integrate the system, and the error tolerance was set to $10^{-12}$. The position vector of the deputy $\mathbf{X}(t)$ can be obtained by directly integrating Eq. (11) with the initial condition of Eq. (63). The result is described in the ECI frame. If the position vector is desired to be described in the Hill frame, Eq. (19) can be used

$$
\left[\begin{array}{l}
x \\
y \\
z
\end{array}\right]=\mathbf{R}\left[\begin{array}{c}
X \\
Y \\
Z
\end{array}\right]-\left[\begin{array}{c}
r_{0} \\
0 \\
0
\end{array}\right]
$$

Next, we simulate the constrained motion. The ODE solver ODE45 in MATLAB was again used to numerically integrate the system, and the error tolerance was set to $10^{-12}$ as before. The position vector of the deputy $\mathbf{X}(t)$ can be obtained by directly integrating Eq. (53) with the initial condition of Eq. (63). Then, we transform the position vector in the ECI frame into that in the Hill frame using Eq. (65).

Fig. 2 shows the trajectory of the deputy projected on the $y z$-plane in the Hill frame without the constraint. The scale is normalized to $\rho$, and the relative motion is unbounded and moving left in the $y$ direction, which shows the necessity of the constraint, say, thrust. This means that the initial conditions given by Eq. (61) fail to keep the desired circular orbit. This is because these initial conditions assume that the linear $\mathrm{HCW}$ equations hold, that is, that the relative distance between the deputy and the chief is small. However, since we are dealing here with the largerelative-distance case $(\rho=50 \mathrm{~km})$, this assumption of linearity no longer holds. In Fig. 3, the constrained motion of the deputy is shown. Also, the scale is normalized to $\rho$. The trajectory is being maintained very well with the relative distance of $\rho=50 \mathrm{~km}$.

Figs. 4 and 5 depict the trajectories of the deputy projected on the $x z$-plane in the Hill frame without and with the constraint, respectively. The scale is normalized to $\rho$ as before. There is not much difference between the trajectories in these two figures. In Figs. 6 and 7 the three-dimensional trajectories in the Hill frame without and with the constraint, respectively, are displayed. It is critical to note that there is a large drift for the unconstrained case in the $y$ direction. 


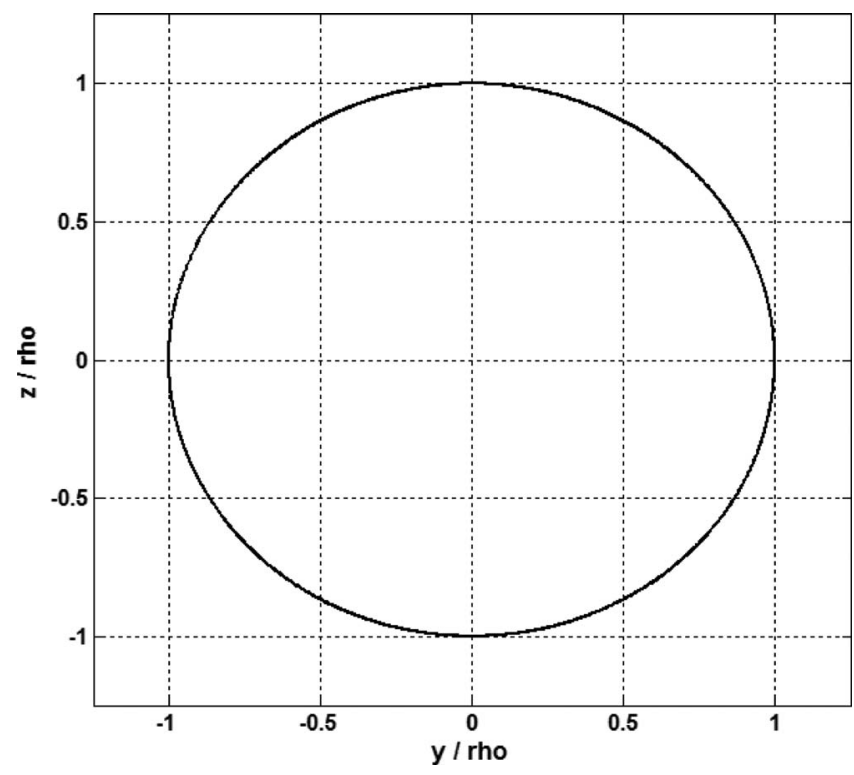

Fig. 3. Constrained motion in the $y z$-plane

In Fig. 8 the required forces to maintain the desired formation and their total magnitude are represented. They are described in the ECI frame and calculated using Eq. (54). The largest thrust is in the $Y$ direction, which dominates the total magnitude, and it corresponds to about $1.3 \mathrm{mN}$ per $\mathrm{kg}$. According to Underwood et al. (2003), the Surrey SNAP-1 nanosatellite whose mass is only $6.5 \mathrm{~kg}$ can deliver $7.7 \mathrm{mN}$ per $\mathrm{kg}$. Therefore, we conclude that all of the thrusts required are attainable by even this small satellite. Fig. 9 shows the differences between the unconstrained and constrained motion for each axis. The displacements for the unconstrained motion are subtracted from the displacements for the constrained motion. The time unit is normalized to the period of the chief. The major drift is observed in the $y$ direction, and this means that without the application of a control force, the deputy satellite will lag behind as time goes by and it would eventually

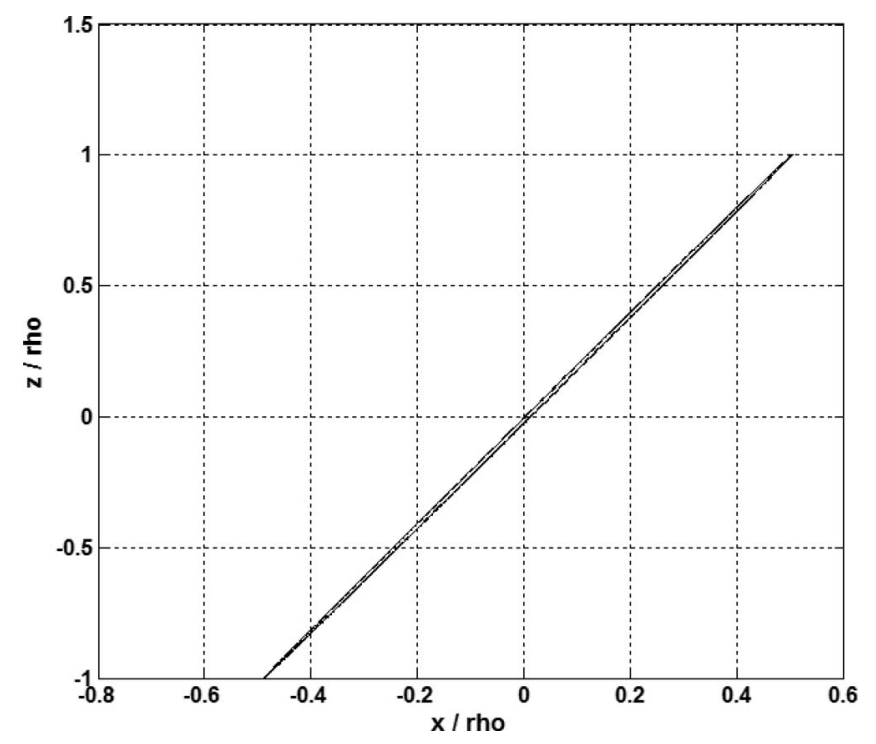

Fig. 4. Unconstrained motion in the $x z$-plane

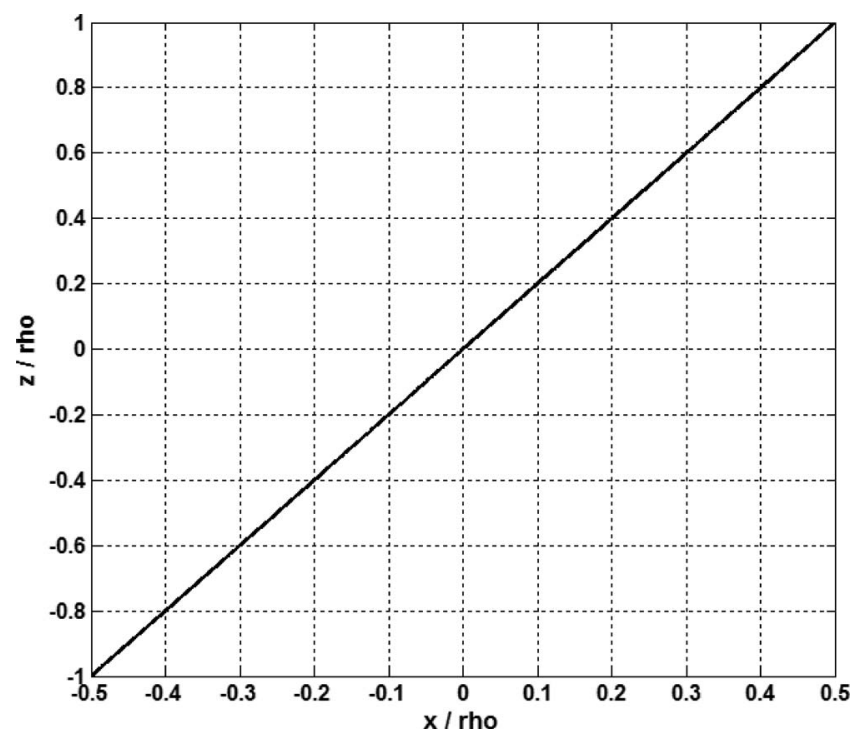

Fig. 5. Constrained motion in the $x z$-plane

fail to maintain the desired station keeping. At the final time, the difference in the $y$ direction approximately corresponds to 0.4 $\times \rho=20 \mathrm{~km}$. Figs. 10-13 represent errors described by Eqs. (57) and (58). The time unit is normalized to the period of the chief as before. The largest displacement error corresponds to about $10^{-4} \mathrm{~m}$ or $0.1 \mathrm{~mm}$. (Fig. 10) This is very small when compared with the relative orbit size $\rho=50 \mathrm{~km}$. Also, the largest velocity error corresponds to only about $3.6 \times 10^{-7} \mathrm{~m} / \mathrm{s}$ (Fig. 12). We see therefore that the errors are so small that the relative orbit is being maintained very well as desired.

\section{Conclusions}

In this paper, a simple, new method for the formation-keeping problem, which is not restricted to the relative size of the formation, is presented. We make use of a recently proposed approach - the Udwadia-Kalaba equation - and this method cap-

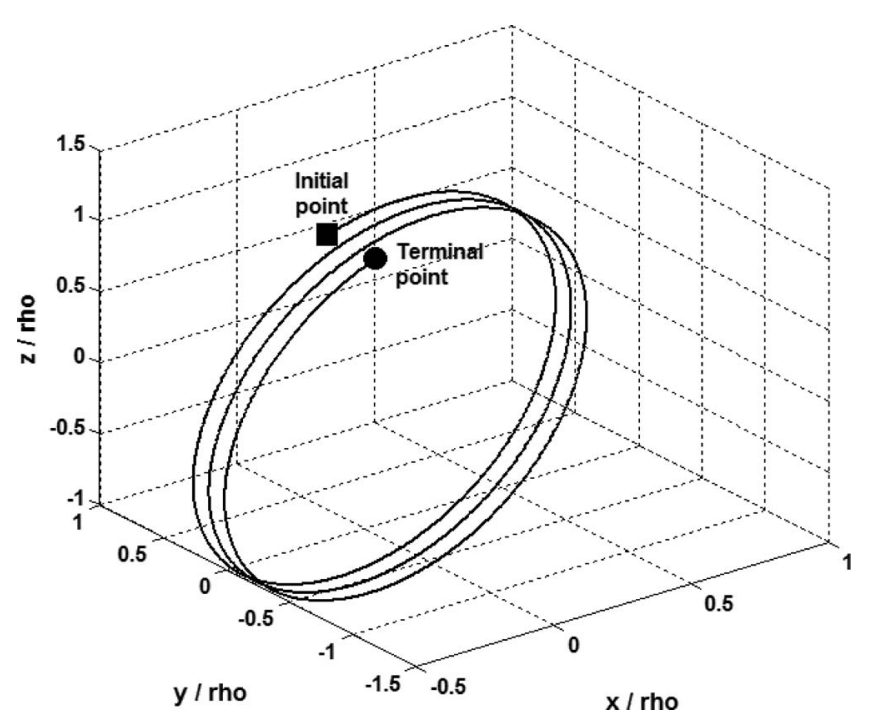

Fig. 6. Unconstrained motion in three dimensional 


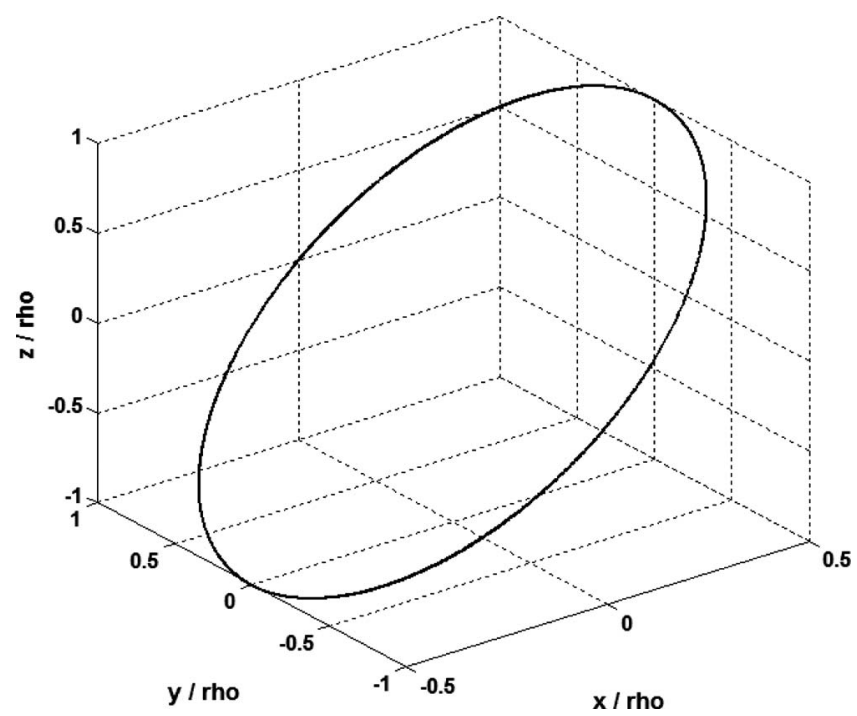

Fig. 7. Constrained motion in three dimensional

tures the complete nonlinearities of the dynamical system. The main conclusions of the paper are the following:

1. We obtain the exact closed-form solution to the problem of formation keeping, something that has not been previously known. This is because the proposed method does not linearize the equations of relative motion. Unlike previous research, we directly start from the nonlinear equation in the ECI frame [Eq. (11)] and the simple exact solution is easily obtained that completely captures all the nonlinearities. We show that the approximate linearized HCW equations will not lead to proper station keeping. With this new approach, however, the constraints are automatically and exactly satisfied, and the exact control function can be explicitly obtained in an easy and straightforward way.

2. While the problem has been solved here for the so-called PCO, this method can be readily generalized to incorporate other types of constraints as well. The strength of this paper
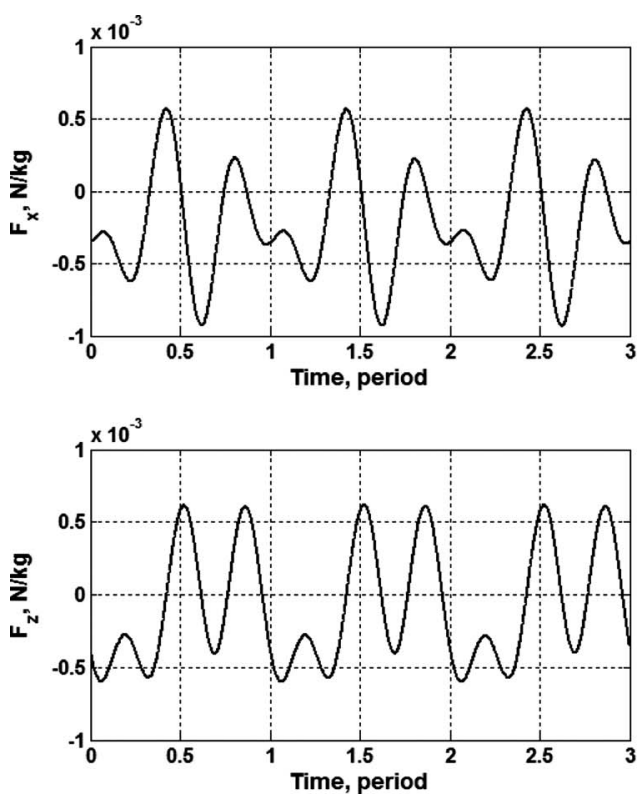

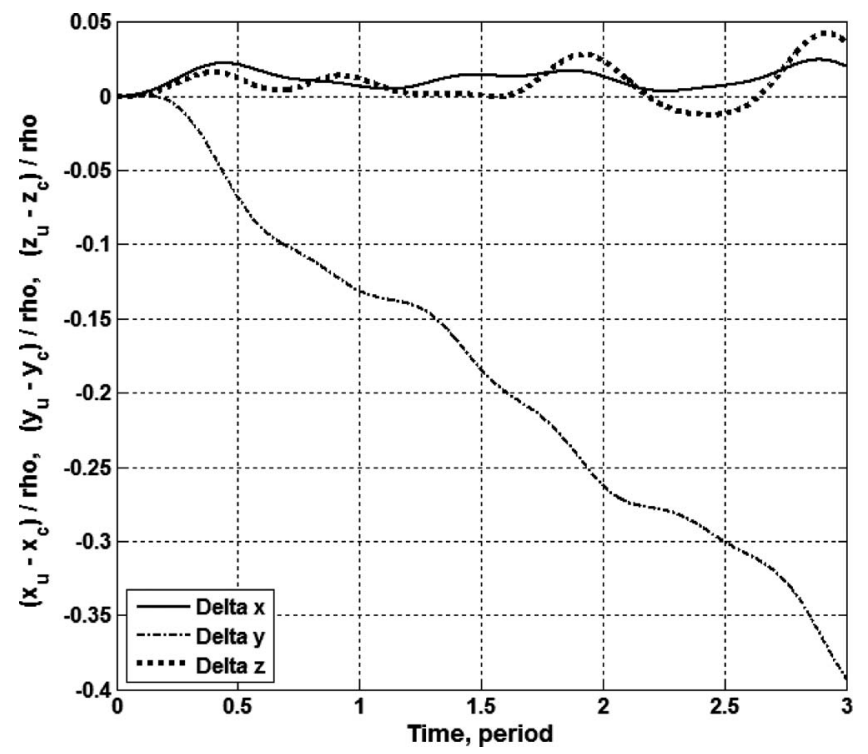

Fig. 9. Differences between the unconstrained and constrained motion

is that once the constraints could be given in a form of Eq. (5), we can analytically solve the highly nonlinear problem in a very easy way. It should be noted again that the Udwadia-Kalaba equation can handle both holonomic and nonholonomic constraints with equal ease.

3. The Udwadia-Kalaba equation in this paper has been described in the inertial frame of reference, and since visualizing the constrained motion in the Hill frame is easier than in the inertial frame, the dynamical motion, after all the calculations are done, is eventually represented in the Hill frame. Each coordinate frame can be easily transformed from the other using Eq. (19), which facilitates the use of the Udwadia-Kalaba equation even when dealing with the problem, which has been traditionally investigated in the Hill frame.
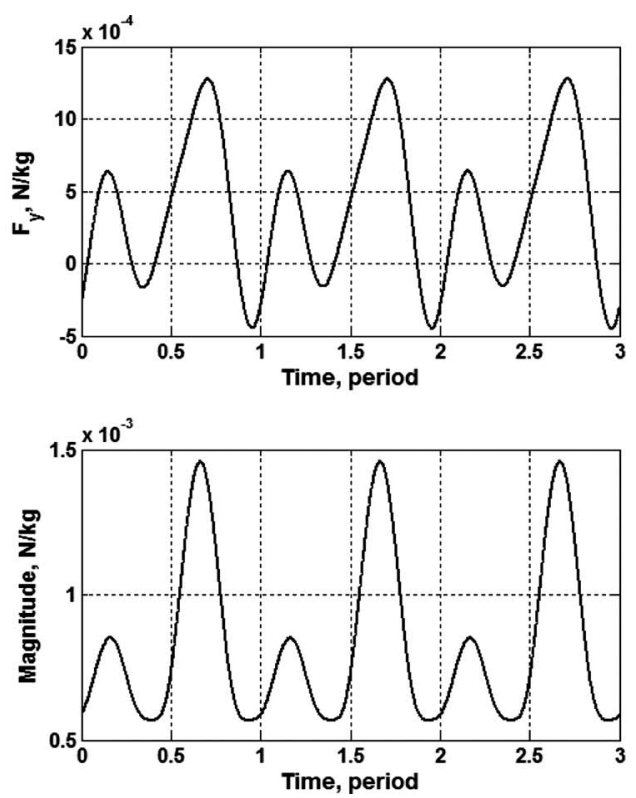

Fig. 8. Required control force in the ECI frame along each axis and its magnitude 


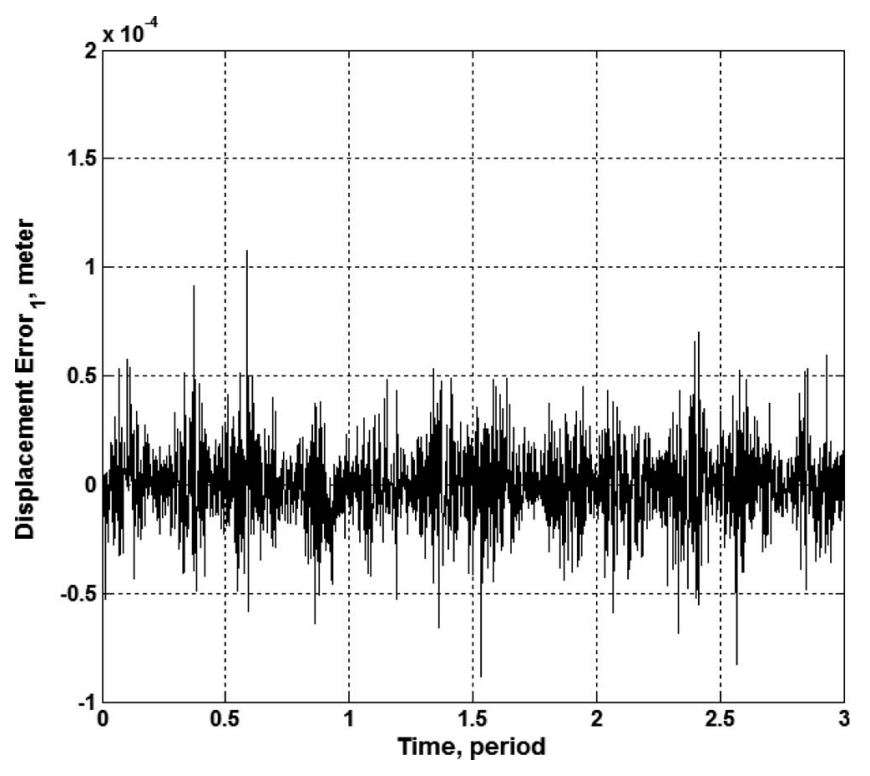

Fig. 10. Displacement error for the first constraint

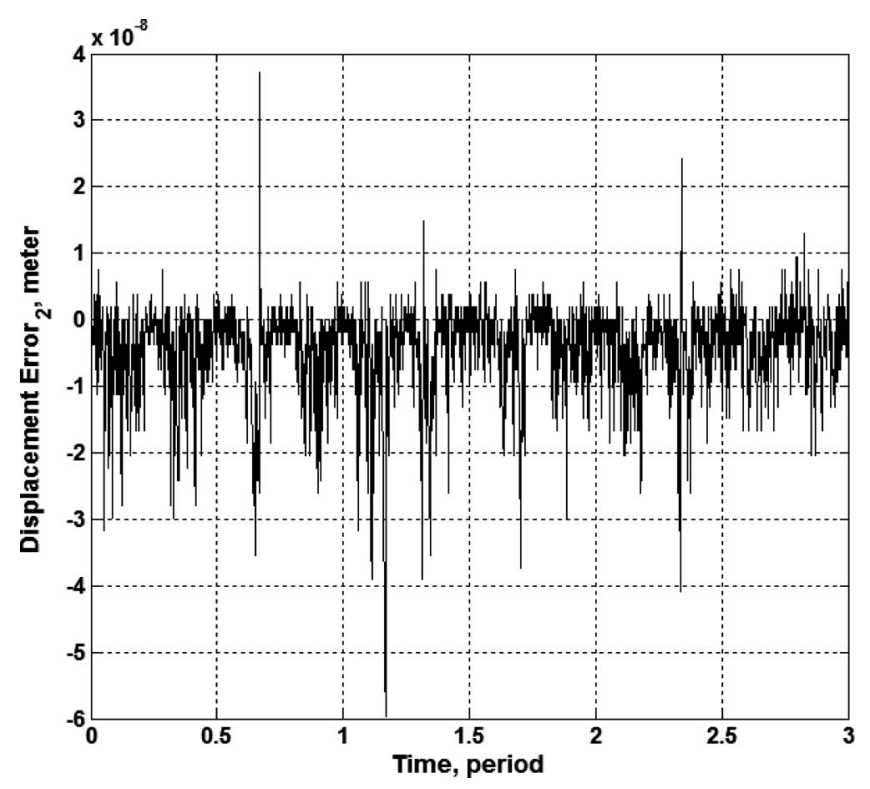

Fig. 11. Displacement error for the second constraint

4. The fact that we have the exact solution to the nonlinear dynamics and control problem is corroborated by the numerical examples that show that the formation is pretty well maintained, as desired, even when the relative distance between the satellites is not small. The errors between the controlled and desired values are extremely small.

5. Finally, we note that the methodology presented in this paper does not include any other perturbations. Future work is planned to consider $J_{2}$ perturbations on the satellites and/or situations where the chief is in a general elliptic (or hyperbolic) Keplerian orbit. Then, we will be able to have a solution to the formation-keeping problem that makes up for all the weak points in the linearized HCW equations.

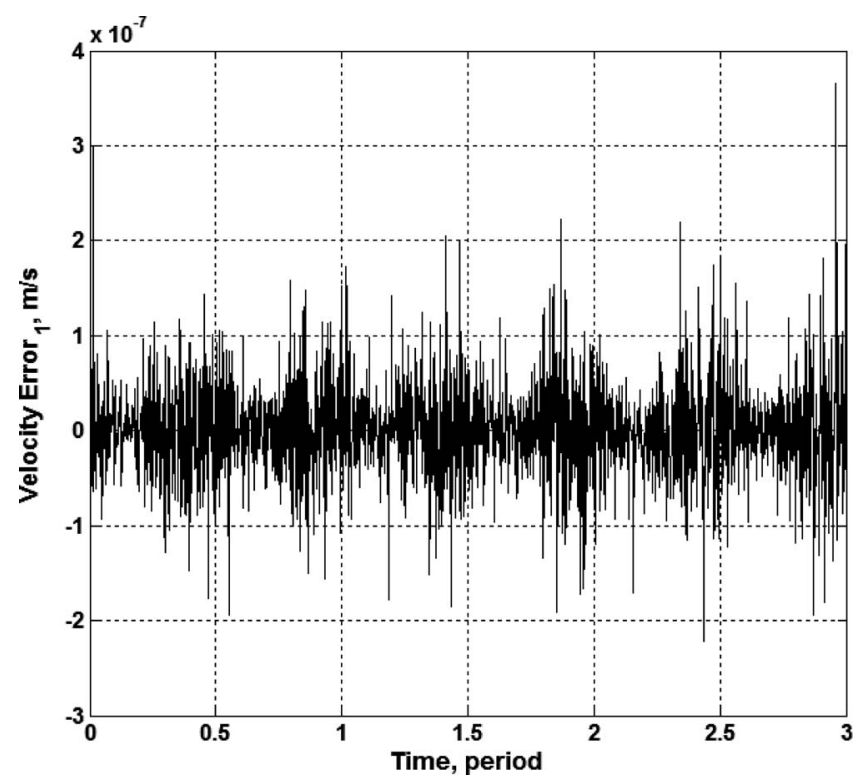

Fig. 12. Velocity error for the first constraint

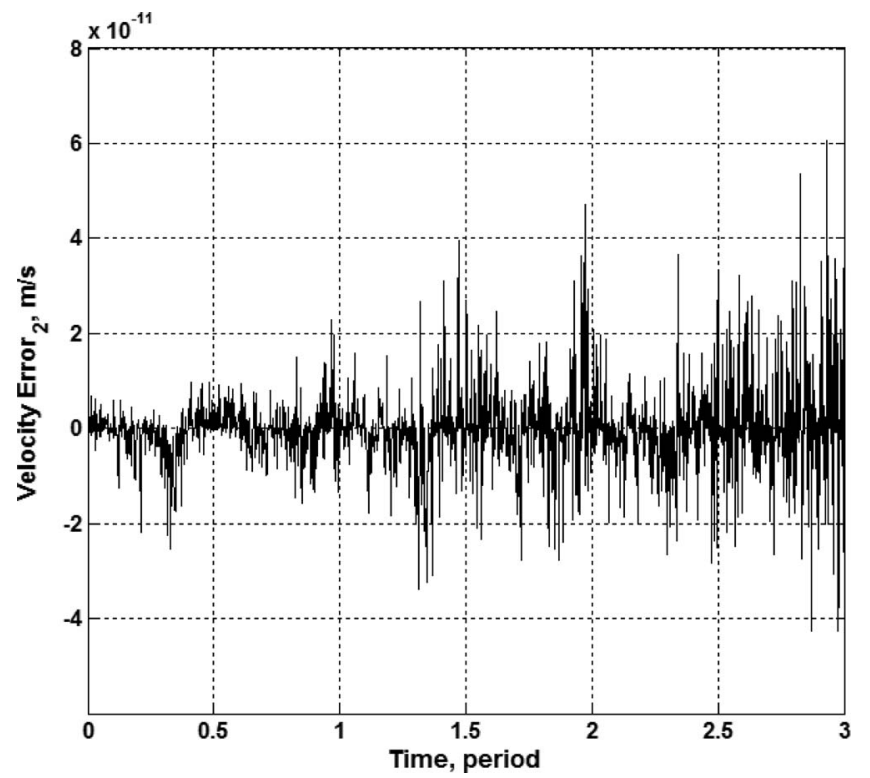

Fig. 13. Velocity error for the second constraint

\section{References}

Armellin, R., Massari, M., and Finzi, A. E. (2004). "Optimal formation flying reconfiguration and station keeping maneuvers using low thrust propulsion." Proc., 18th Int. Symp. on Space Flight Dynamics (ESA SP-548), ESA Publication Division, Noordwijk, 429-434.

Clohessy, W. H., and Wiltshire, R. S. (1960). "Terminal guidance system for satellite rendezvous.” J. Aerosp. Sci., 27(9), 653-658, 674.

Hill, G. W. (1878). "Researches in the lunar theory." Am. J. Math., 1(1), $5-26$.

Kalaba, R. E., and Udwadia, F. E. (1993). "Equations of motion for nonholonomic, constrained dynamical systems via Gauss's principle." J. Appl. Mech., 60(3), 662-668.

Karlgaard, C. D., and Lutze, F. H. (2003). "Second-order relative motion equations." J. Guid. Control Dyn., 26(1), 41-49.

Kasdin, N. J., Gurfil, P., and Kolemen, E. (2005). "Canonical modelling of relative spacecraft motion via epicyclic orbital elements." Celest. 
Mech. Dyn. Astron., 92(4), 337-370.

Peters, J., Mistry, M., Udwadia, F. E., and Schaal, S. (2005). "A new methodology for robot controller design." Proc., 5th ASME Int. Design Engineering Technical Conf. and Computers and Information in Engineering Conf. (DETC 2005-95166, Vol. 5, ASME, 1-10.

Prussing, J. E., and Conway, B. A. (1993). Orbital mechanics, Oxford University Press, New York.

Qingsong, M., Pengji, W., and Di, Y. (2004). "Low-thrust fuzzy formation keeping for multiple spacecraft flying." Acta Astronaut., 55(11), 895-901.

Richardson, D. L., and Mitchell, J. W. (2003). "A third-order analytical solution for relative motion with a circular reference orbit." J. Astronaut. Sci., 51(1), 1-12.

Sabol, C., Burns, R., and McLaughlin, C. A. (2001). "Satellite formation flying design and evolution.” J. Spacecr. Rockets, 38(2), 270-278.

Schutte, A. D., and Dooley, B. A. (2005). "Constrained motion of tethered satellites." J. Aerosp. Eng., 18(4), 242-250.

Sparks, A. (2000). "Satellite formation keeping control in the presence of gravity perturbations." Proc., American Control Conf., Vol. 2, Institute of Electrical and Electronics Engineering, 844-848.

Udwadia, F. E. (2000). "Nonideal constraints and Lagrangian dynamics." J. Aerosp. Eng., 13(1), 17-22.

Udwadia, F. E. (2002). "Equations of motion for mechanical systems: A unified approach." Int. J. Nonlinear Mech., 69, 951-958.

Udwadia, F. E. (2003). "A new perspective on the tracking control of nonlinear structural and mechanical systems." Proc. R. Soc. London,
Ser. A, 459, 1783-1800.

Udwadia, F. E. (2005). "Equations of motion for constrained multibody systems and their control." J. Optim. Theory Appl., 127(3), 627-638.

Udwadia, F. E. (2008). "Optimal tracking control of nonlinear dynamical systems." Proc. R. Soc. London, Ser. A, 464, 2341-2363.

Udwadia, F. E., and Kalaba, R. E. (1992). "A new perspective on constrained motion." Proc. R. Soc. London, Ser. A, 439, 407-410.

Udwadia, F. E., and Kalaba, R. E. (1999). "A unified approach for the recursive determination of generalized inverses." Comput. Math. Appl., 37(1), 125-130.

Udwadia, F. E., and Kalaba, R. E. (2002). "What is the general form of the explicit equations of motion for constrained mechanical systems." J. Appl. Mech., 69, 335-339.

Udwadia, F. E., and Kalaba, R. E. (2008). Analytical dynamics: A new approach, Cambridge University Press, New York.

Underwood, C. I., Richardson, G., and Savignol, J. (2003). "In-orbit results from the SNAP-1 nano-satellite and its future potential." Philos. Trans. R. Soc. London, Ser. A, 361, 199-203.

Vaddi, S. S., Vadali, S. R., and Alfriend, K. T. (2003). "Formation flying: Accommodating nonlinearity and eccentricity perturbations." J. Guid. Control Dyn., 26(2), 214-223.

Yan, Q., Kapila, V., and Sparks, A. G. (2000). "Pulse-based periodic control for spacecraft formation flying." Proc., American Control Conf., Vol 1, Institute of Electrical and Electronics Engineering, 374378. 